\title{
Aged IRF3-KO Mice are Protected from Sepsis
}

\author{
Dinesh G Goswami' \\ Wendy E Walker (D) ${ }^{1,2}$ \\ 'Center of Emphasis in Infectious \\ Diseases, Department of Molecular and \\ Translational Medicine, Paul L. Foster \\ School of Medicine, Texas Tech \\ University Health Sciences Center EI \\ Paso, El Paso, TX, USA; ${ }^{2}$ Graduate \\ School of Biomedical Sciences, Texas \\ Tech University Health Sciences Center \\ El Paso, El Paso, TX, USA
}

Purpose: Sepsis is a leading cause of hospital admissions and deaths. Older adults $(>65$ years) are particularly susceptible to sepsis and experience higher morbidity and mortality rates than younger people. We previously showed that interferon regulatory factor 3 (IRF3) contributes to sepsis pathogenesis in young mice subject to cecal ligation and puncture (CLP). In this study, we investigated if IRF3 contributes to sepsis in the context of aging.

Methods: Sepsis was induced in aged wild-type (WT) and IRF3-knock-out (KO) mice, using a clinically-relevant CLP-sepsis model including fluids and antibiotics. Animal survival, disease score and hypothermia were evaluated as indicators of sepsis pathogenesis. Serum cytokines and serum enzymes indicative of organ damage were also measured.

Results: Aged WT mice were highly susceptible to sepsis (90\% mortality). In comparison, aged IRF3-KO mice were significantly protected (20\% mortality). Aged IRF3-KO mice showed a lower disease score and reduced hypothermia following CLP, compared to WT mice. Serum cytokines interleukin (IL)-6, IL-12/23p40 and macrophage chemoattractant protein (MCP)-1, and creatinine kinase (CK) were lower in aged IRF3-KO septic mice compared to WT counterparts. Aged male mice were found to be more susceptible to sepsis compared to females. Female mice, however, produced higher levels of serum cytokines and CK.

Conclusion: These results demonstrate that IRF3 plays a detrimental role in sepsis in aged mice and highlight the impact of biological sex.

Keywords: septicemia, CLP, interferon regulatory factor 3, aging, mice, sex

\section{Introduction}

Sepsis is a life threatening condition defined by immune dysregulation and organ dysfunction secondary to an infection. Although sepsis can affect anyone who develops an infection, certain groups have an increased risk of developing sepsis, including infants, older people (seniors) and individuals with comorbidities such as diabetes and cancer. ${ }^{1}$ Of relevance to this study, sepsis is particularly detrimental for older people, with higher incidence ( $60 \%$ of people diagnosed are $>65$ years old) and increased mortality rates $(80 \%$ of all septic deaths are in people $>65$ years old). ${ }^{2,3}$ In addition, sepsis is responsible for the most expensive hospital stays worldwide, incurring a huge financial burden. ${ }^{4}$ Even for patients who are successfully discharged, the chances of hospital readmission are high and there is frequently an overall decrease in the quality of life, especially amongst older sepsis survivors. ${ }^{5-7}$ Older patients exhibit a host of immune defects that include both qualitative and quantitative changes in the innate and adaptive immune response, ${ }^{8}$ compromising their ability to fight infections. Furthermore, as people age, they experience an increase in comorbidities (such as diabetes and hypertension), ${ }^{9}$ contributing to the higher sepsis incidence and mortality. Advancements in sepsis care and early intervention with antibiotics and vasopressors have improved sepsis
Correspondence: Wendy E Walker Texas Tech University Health Sciences Center El Paso, 500I El Paso Drive, El Paso, TX, 79905, USA

Tel $+|-9| 5-2 \mid 5-4268$

Fax + I-915- 783-1253

Email wendy.walker@ttuhsc.edu 
mortality rates. Nevertheless, understanding how sepsis affects older adults continues to be of great importance.

Due to their short life span, mice are a convenient model for aging research. Studies have shown that aged mice experience increased sepsis mortality relative to young adult counterparts in both surgical and non-surgical models of sepsis. ${ }^{3,10-13}$ This has been attributed to higher levels of cytokines with an inability to return to baseline, increased splenic apoptosis, ineffective or sub-optimal protective immunity, and insufficient myelopoiesis. ${ }^{3,10,11}$

Interferon regulatory factor 3 (IRF3) is a transcription factor that is activated downstream of multiple pattern recognition pathways (PRRs). ${ }^{14-18}$ A large number of PRRs activate IRF3 including: Toll-like Receptors (TLRs) that act via TIR-Domain Containing Adapter-inducing Interferon- $\beta$ (TRIF), ${ }^{14}$ cytosolic Cyclic GMP-AMP synthase (cGAS) pathway that acts via Stimulator of Interferon Genes (STING), ${ }^{15}$ the Retinoic acid-inducible gene I (RIG-I)-like receptors (RLRs) that signal via Mitochondrial antiviral-signaling protein (MAVS), ${ }^{16}$ the Nucleotide-binding oligomerization domain 2 (NOD2) receptor pathway, ${ }^{17}$ and the Absent in melanoma 2 (AIM2), ${ }^{18}$ amongst others. These PRRs recognize pathogen associated molecular patterns (PAMPs), such as lipopolysaccharide and dsRNA, and damage associated molecular patterns (DAMPSs) such as cell-free serum DNA, and subsequently initiate a signal transduction cascade. This culminates in phosphorylation of IRF3, which induces protein dimerization and translocation into the nucleus. IRF3 then induces the expression of its classical target Interferon (IFN) $\beta$, as well as a number of InterferonStimulated genes (ISGs). These mediators improve host defense against viral infections, but may also increase inflammation with both beneficial and harmful downstream effects.

Previous reports from our lab showed that young adult IRF3-KO mice are significantly protected from sepsis induced by CLP in the absence ${ }^{19}$ or presence ${ }^{20}$ of clinical treatments. Absence of IRF3 was also found to protect mice from the systemic inflammatory response induced by liposome:DNA. ${ }^{21}$ IRF3 can be activated by multiple pattern recognition receptors. ${ }^{22,23}$ In the CLP model we have shown TIR-Domain Containing Adapter-inducing Interferon- $\beta$ (TRIF) and Stimulator of Interferon Genes (STING) activate IRF3 and contribute to sepsis pathogenesis, depending on the model employed. ${ }^{24}$ Further, in our recently published report we showed that IRF3 acts in the stromal (non-leukocyte) compartment to alter the inflammatory network during sepsis. ${ }^{20}$ Surprisingly, we did not detect IFN $\beta$ in the blood or peritoneal lavage of septic mice, ${ }^{20}$ suggesting that IRF3 may act via an IFNindependent mechanism to exacerbate sepsis.

In the present study, we hypothesized that IRF3 also contributes to sepsis pathogenesis in the context of aging. ${ }^{19,20}$ To study this, we utilized a clinically relevant model of sepsis induced by CLP, incorporating crystalloid fluids and antibiotics. We found that aged IRF3-KO mice are protected from sepsis in comparison to their WT counterparts. These data indicate that IRF3 plays a detrimental role in sepsis in the context of aging, and could represent a therapeutic target for sepsis treatment.

\section{Materials and Methods}

\section{Animals}

All the animal procedures carried out in this study were approved by the Institutional Animal Care and Use committee at TTUHSC El Paso. The research complied with the PHS Policy on Humane Care and Use of Laboratory Animals, and followed the recommendations presented in "The Guide for the Care and Use of Laboratory Animals". ${ }^{25}$ C57BL/6J (Jackson labs) and IRF3-KO mice (obtained from Yale University) were bred in the American Association for Accreditation of Laboratory Animal Care (AAALC)-accredited vivarium at the Laboratory Animal Resource Center at TTUHSC at El Paso in clean Optimice cages (Animal Care Systems, Centennial, CO) and aged within our animal facility. The animals were supplied with corncob bedding, nestlets, and polycarbonate huts as enrichment. The mice were housed in social groups (2-5 mice per cage; with individual housing in rare cases only) under a $12 \mathrm{~h}$ light $/ 12 \mathrm{~h}$ dark cycle. The animals had ad libitum access to water and food (5R53 Extruded PicoLab Rodent Diet 20, Lab Diet, St. Louis, MO). The pathogen-free status of the mice was ensured by regular testing of sentinel mice for specific pathogens (including helicobacter and norovirus). The study included a total of 25 mixed gender aged mice (16.5-20 months old), including $\mathrm{C} 57 \mathrm{BL} / 6 \mathrm{~J}$ mice ( $\mathrm{n}=10$ subject to CLP, $50 \%$ female and $n=5$ subject to sham surgery, $40 \%$ female) and IRF3-KO mice ( $\mathrm{n}=10$ subject to CLP, $50 \%$ female). Please note, these animal numbers reflect the sum of 5 experimental repeats, and pooled data for the entire cohort are shown in the results. 


\section{Cecal Ligation and Puncture (CLP)}

CLP surgeries were performed per our prior reports. ${ }^{20,24}$ Briefly, mice were anesthetized with isoflurane (2-2.5\%) using a precision vaporizer. Buprenorphine-SR $(1 \mathrm{mg} / \mathrm{kg}$; Zoopharm, Windsor, $\mathrm{CO}$ ) was given s.c. prior to the surgery for pain management. The abdomen was shaved and scrubbed with betadine and ethanol in succession (thrice). A surgical incision was made in the abdomen and cecum was externalized. $1 \mathrm{~cm}$ of the cecum was ligated with a silk suture and the cecum was punctured through and through using a $21 \mathrm{G}$ needle. A small drop of the cecal contents was extruded and the cecum was placed back in the abdominal cavity. The peritoneum was closed with a running suture and the skin was closed by interrupted sutures (both layers used 5-0 coated Vicryl violet braid suture, Ethicon, Somerville, NJ). Sham surgeries were performed similarly, except the cecum was not ligated and not punctured. All surgeries were carried out between 1-4 pm. $1 \mathrm{~mL}$ lactated ringer's solution was administered s.c. post-op. The mice received $25 \mathrm{mg} / \mathrm{kg}$ imipenem/cilastatin (Primaxin, Merck, Whitehouse Station, NJ) i.p. at $6 \mathrm{~h}$ post-surgery and then twice daily (9-10am in the morning and 5-6pm in the evening) for a total of 8 doses. For mice showing continued signs of pain and discomfort, additional doses of buprenorphine-SR were administered at $48-72 \mathrm{~h}$ intervals. Please note that this model follows the recent recommendations for Minimum Quality Threshold in Pre-Clinical Sepsis Studies (MQTiPSS). ${ }^{26}$

\section{Measurement of Sepsis Disease Score, Temperature, Serum Cytokines, CK and ALT}

Following CLP, the mice were checked three times daily and their survival was monitored. Inability to regain sternal recumbence was utilized as a humane endpoint; these animals were euthanized and scored as "dead" (with the time of death assigned at the subsequent observational time point). Mice were scored for the disease as described previously, ${ }^{19}$ according to their degree of lethargy (0-alert, 1-slightly lethargic, 2-lethargic, 3-very lethargic, 4-dead). The body surface temperature of the mice was measured (on the sternum) using an infrared thermometer (Traceable Temptestr Mini Infrared Thermometer, Cole-Parmer, Vernon Hills, IL, USA). The mice were bled before surgery $(0 \mathrm{~h})$ and at 6 and 18-h post-surgery via the retro-orbital plexus, as previously described, ${ }^{27}$ and serum was prepared using gold-top microtainers (Becton Dickinson, Franklin
Lakes, NJ), per the manufacturer's instructions. We selected the $0 \mathrm{~h}$ (baseline), $6 \mathrm{~h}$ and $18 \mathrm{~h}$ time points because our prior experiments indicate that serum cytokines are substantially elevated at $6 \mathrm{~h}$ and $18 \mathrm{~h}$ post-sepsis. ${ }^{19,20,24,28}$ IL- 6 and IL-12/ $23 \mathrm{p} 40$, and MCP-1 were quantified in the serum using BD OptEIA ELISA kits (Becton Dickinson, Franklin Lakes, NJ). The CK and Alanine Aminotransferase (ALT) enzymes were measured in serum samples using the $\mathrm{CK}$ Liquid Reagent Set, and ALT Liquid Reagent Set (both from Pointe Scientific Inc. MI, USA), per the manufacturer's protocol.

\section{Statistical Analyses}

Statistical analyses were carried out using Prism 6 (GraphPad, San Diego, CA). The experiments were repeated 4 times and the graphs show the pooled results. Kaplan-Meier Log rank test was used to compare the survival curves. Two-way repeated measures ANOVA was used to compare the mouse surface temperature and disease score. Cytokine data were compared using Mann Whitney tests at 6 and 18h (the WT and IRF3-KO CLP groups were compared). $P$ values $<0.05$ were considered statistically significant.

\section{Results}

\section{Aged IRF3-KO Mice are Protected from Sepsis}

In this study, we sought to determine if IRF3 exacerbates sepsis in the context of aging. To address this, we induced sepsis via CLP in aged WT mice $(n=10$, average age: 82 weeks, SD: 4.5, 50\% females) and aged IRF3-KO mice ( $\mathrm{n}=10$, average age: 82 weeks, SD: 4.3, 50\% females). Sham WT controls $(\mathrm{n}=5$, all aged 71.86 weeks, $40 \%$ females) were included to identify specific effects of sepsis vs surgery. All animals were administered lactated ringer's solution post-op and imipenem/cilastatin antibiotics at $6 \mathrm{~h}$ post-surgery and then twice daily.

We found that aged WT mice were highly susceptible to sepsis, exhibiting 90\% mortality (Figure 1A). In comparison, aged IRF3-KO mice were substantially protected (20\% mortality, Figure 1A). The aged WT mice had a significantly higher disease score (eg lethargy; Figure 1B) and developed more severe hypothermia, (Figure 1C) compared to the aged IRF3-KO mice. All of the WT sham mice survived (Figure 1A) and remained alert (Figure 1B) and none showed signs of hypothermia (Figure 1C). 


\section{Pooled}

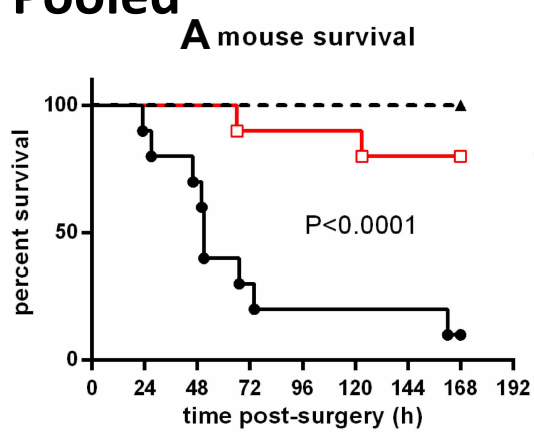

Male

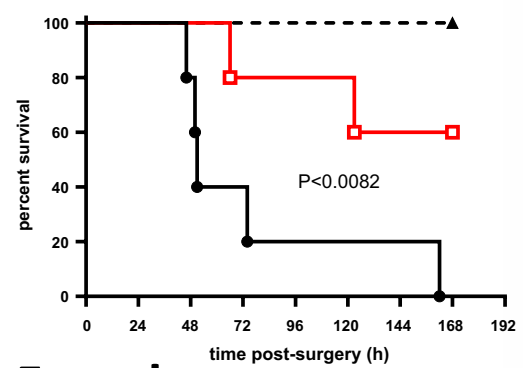

Female

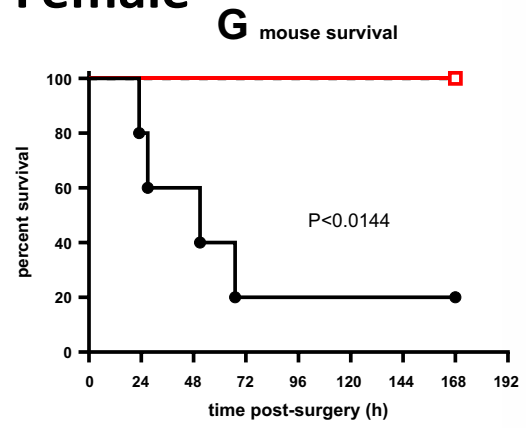

B disease score

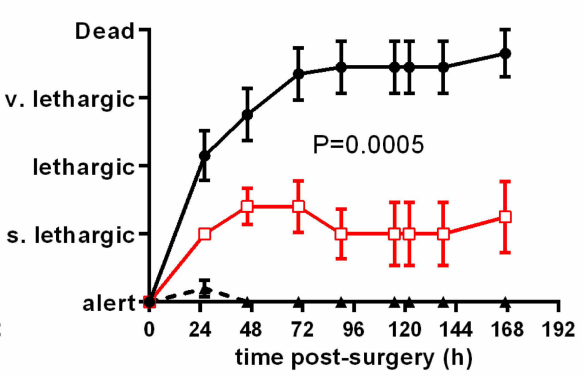

E disease score

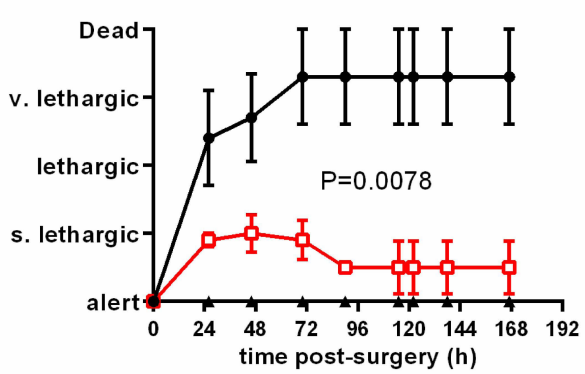

$H$ disease score

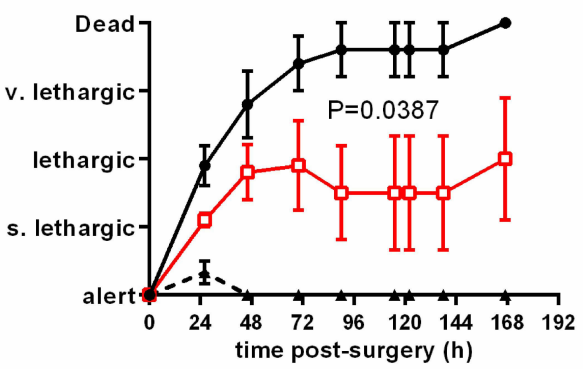

C surface temperature

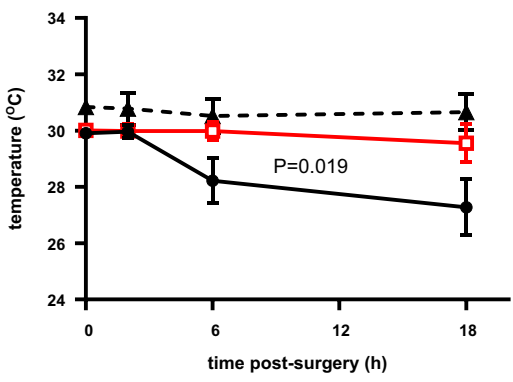

$F_{\text {surfacete mperature }}$

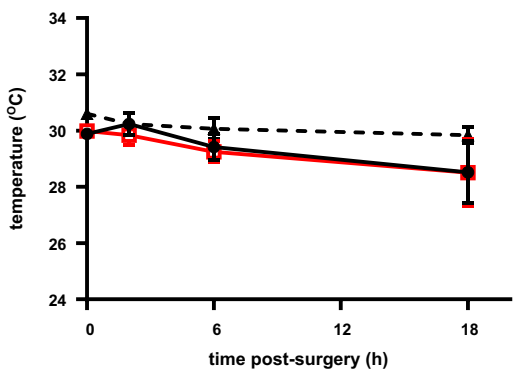

I surface temperature

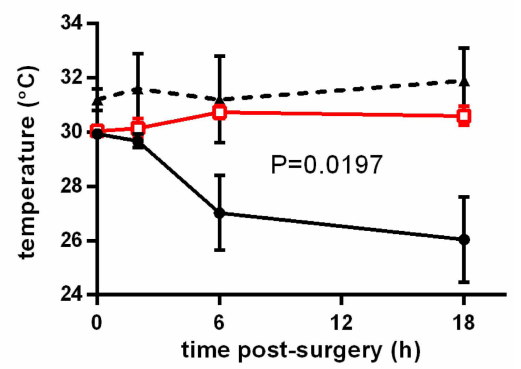

$\begin{array}{ll}-\bullet \cdot & \text { WT sham } \\ \rightarrow & \text { WT CLP } \\ \rightarrow- & \text { IRF3-KO CLP }\end{array}$

Figure I Aged IRF3-KO mice were protected from sepsis. Aged WT and IRF3-KO mice were subject to CLP to induce sepsis, and a second group of aged WT mice was subject to sham surgery as a control. Graphs show pooled male and female data $(n=10 /$ group CLP, $n=5 /$ group sham) for (A) animal survival, (B) disease score (degree of lethargy) and (C) surface temperature, indicative of hypothermia. This cohort was split into male and female subgroups to show the impact of biological sex. Graphs show data for the male mice

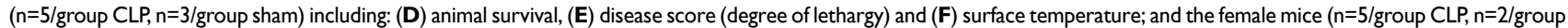
sham), including: (G) animal survival, (H) disease score (degree of lethargy) and (I) surface temperature. P values show the results of a log rank test comparing the WT and IRF3-KO CLP groups for animal survival and two-way repeated measures ANOVA comparing the WT and IRF3-KO CLP groups for the other measurements.

We observed a trend toward a higher mortality rate in aged WT males (100\% mortality, Figure 1D) as compared to aged WT females ( $80 \%$ mortality, Figure $1 \mathrm{G})$. Examining the mortality curve more closely, however, the female WT mice exhibited more rapid mortality than males during the acute phase of sepsis (days 0-3), as two females died by $24 \mathrm{~h}$ post-CLP (Figure $1 \mathrm{G}$ ) whereas in the males, the first death occurred at 46h (Figure 1D). In both male WT mice and female WT mice, the median survival time was $51 \mathrm{~h}$. However, the male WT mice exhibited a greater incidence of mortality in the chronic phase, as one additional male animal succumbed at $123 \mathrm{~h}$ post-CLP, resulting in a higher overall mortality rate in WT males vs females.

We also examined the impact of IRF3 specifically within the male and female subcohorts. While all of the male WT mice succumbed to sepsis, the mortality rate was only $40 \%$ in the male IRF3-KO mice (Figure 1D). While 
$80 \%$ of the aged WT female mice succumbed to sepsis, all of the aged female IRF3-KO mice survived (Figure 1G). In both males and females, the WT mice had a higher disease score as compared to the IRF3-KO mice (Figure 1E and $\mathrm{H}$ ). The difference in body temperature was pronounced and significant in female WT mice compared to their IRF3-KO counterparts subject to CLP (Figure 1I). In contrast, male WT mice showed only a slight drop in body temperature after CLP, and there was no difference between the genotypes (Figure 1F).

\section{Aged IRF3-KO Mice Show an Attenuated Serum Cytokine Response Following CLP}

Next, we examined the systemic inflammatory response in these aged IRF3-KO vs WT septic mice. Serum samples were obtained at $0 \mathrm{~h}, 6 \mathrm{~h}$ and $18 \mathrm{~h}$ post-surgery and the cytokines IL-6, IL-12/23p40 and MCP-1 were quantified via ELISA. In aged WT mice, serum levels of IL-6 increased substantially at $6 \mathrm{~h}$ post-CLP surgery and showed a further increase at $18 \mathrm{~h}$ (Figure 2A). In comparison, this cytokine showed minimal elevations in aged IRF3-KO mice subject to CLP (Figure 2A). IL-6 was significantly higher in WT mice compared to IRF3-KO mice at $6 \mathrm{~h}$ and $18 \mathrm{~h}$ post-CLP (Figure 2A). In both WT and IRF3-KO mice, serum levels of IL-12/23p40 peaked at $6 \mathrm{~h}$ postCLP, followed by a decrease at $18 \mathrm{~h}$ (Figure 2B). The levels of serum IL-12/23p40 were higher in WT mice compared to IRF3-KO mice at each time point, but the difference was not statistically significant (Figure 2B).

In aged WT mice, the chemokine MCP-1 increased at $6 \mathrm{~h}$ post-CLP and showed a further increase at $18 \mathrm{~h}$ (Figure 2C). In contrast, in IRF3-KO mice, serum MCP1 increased at $6 \mathrm{~h}$ but then decreased at $18 \mathrm{~h}$ (Figure 2C). The levels of MCP-1 were significantly higher in WT mice compared to IRF3-KO mice at both time points (Figure 2C). Sham operated WT mice produced negligible amounts of serum IL-6, IL-12/23p40 and MCP-1 (Figure 2A-C). Interestingly, aged male WT mice showed a trend towards lower levels of serum cytokines (Figure 2D-F) compared to their female counterparts (Figure 2G-I). Significant difference in IL-6 levels were observed between aged female WT vs IRF3-KO mice at both $6 \mathrm{~h}$ and $18 \mathrm{~h}$ (Figure 2G), while for the male mice significant difference in IL-6 levels were observed at only $18 \mathrm{~h}$ post-CLP (Figure 2D). For IL-12 and MCP-1, the differences between aged WT vs IRF3-KO were more pronounced in female mice (Figure $2 \mathrm{H}$ and I), as compared to their male counterparts (Figure 2E and F).

\section{Aged IRF3-KO Mice Have Reduced Serum CK Levels Following CLP}

Next, we looked for signs of organ damage in these aged IRF3-KO vs WT septic mice. Elevated serum $\mathrm{CK}$ is a sign of heart or muscle damage, and elevated ALT is a sign of liver damage. We previously observed that both CK and ALT were elevated in the sera of young adult septic mice. ${ }^{19} \mathrm{CK}$ levels were reduced in young IRF3-KO vs WT septic mice, while ALT levels were similar between the genotypes. $^{19}$

$\mathrm{CK}$ and ALT were measured in serum samples obtained from aged WT and IRF3-KO at $18 \mathrm{~h}$ post- CLP or sham surgery. Compared to aged sham mice, aged CLP mice had elevated serum CK levels (Figure 3A) and ALT levels (Figure 3B). Among the pooled male and female aged cohort subject to CLP, IRF3-KO mice had significantly lower serum CK levels compared to WT mice (Figure 3A), however their ALT levels were similar (Figure 3B). Interestingly, male WT mice subject to CLP had lower $\mathrm{CK}$ values, but higher ALT values relative to their female counterparts (Figure 3C-F). Among the male mice, we observed a clear trend towards decreased $\mathrm{CK}$ in IRF3-KO mice vs WT mice subject to CLP (Figure 3C), but this difference was not statistically significant. ALT levels were similar in male WT and IRF3-KO mice (Figure 3D). Among the female mice, we observed significantly lower serum CK levels in IRF3-KO mice compared to WT mice subject to CLP (Figure 3E). Female mice exhibited similar ALT levels regardless of their surgery (CLP vs sham) or genotype (WT vs IRF3-KO) (Figure 3F).

\section{Discussion}

It is well known that sepsis is more detrimental in older people vs younger adults. ${ }^{2,3,29-35}$ The underlying mechanism, however, is not completely understood. Mice are a useful model species to study the pathogenesis of sepsis. Aged mice are more susceptible to endotoxin-induced systemic inflammation, and compared to young mice they are more likely to succumb to systemic infection in pneumonia models and from peritonitis following CLP. $^{3,10,12,36-40}$ Aged rodents demonstrate specific defects in the leukocyte response to infection, as well as increased systemic cytokines. $^{32,41}$ Additionally, an inability of the 
Pooled

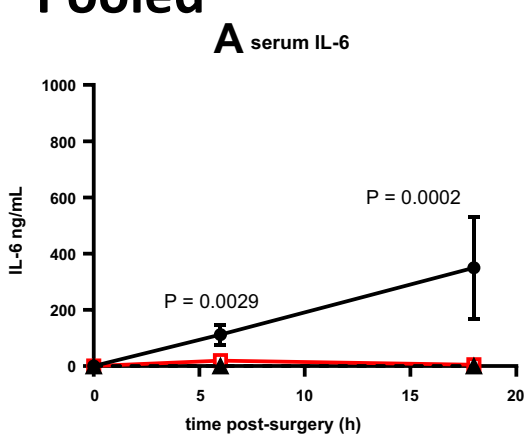

Male D serum LL-6

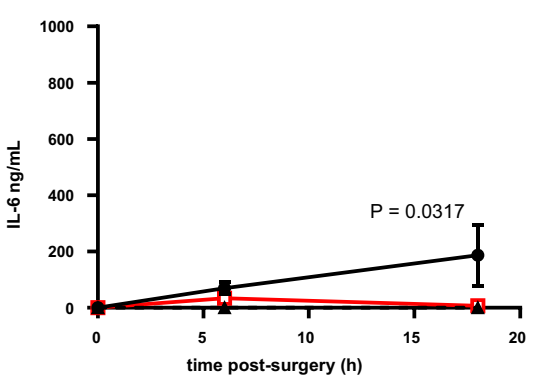

Female

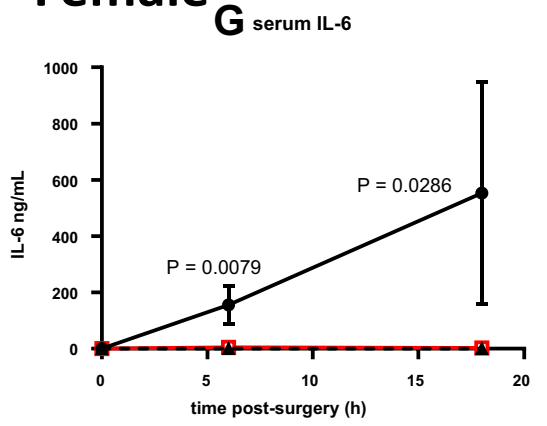

B serum LL-12/23 p 40

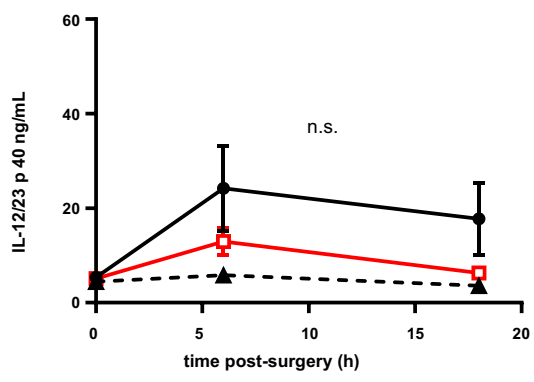

$E_{\text {serum LL-12/23 p } 40}$

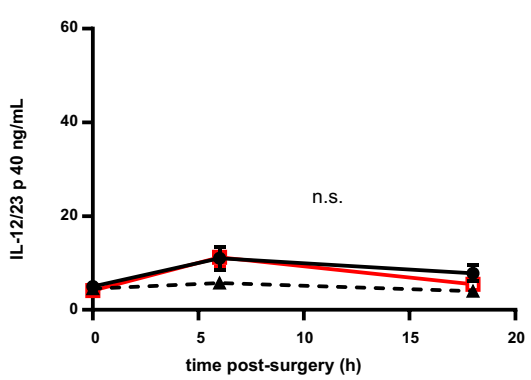

H serum LL-12/23 p 40

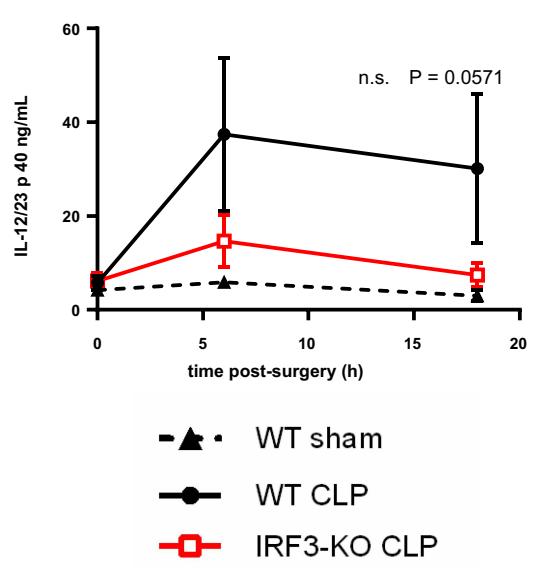

C serum MCP-1

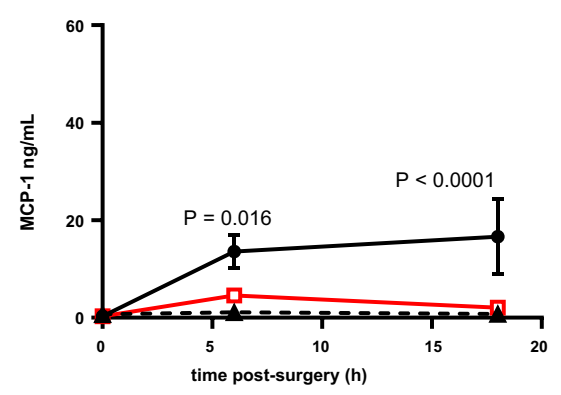

$F_{\text {serum MCP-1 }}$

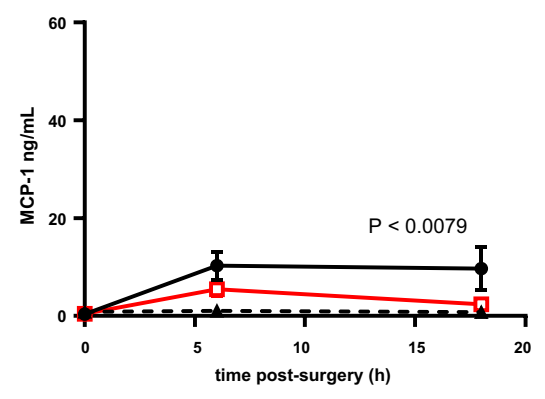

I serum MCP-1

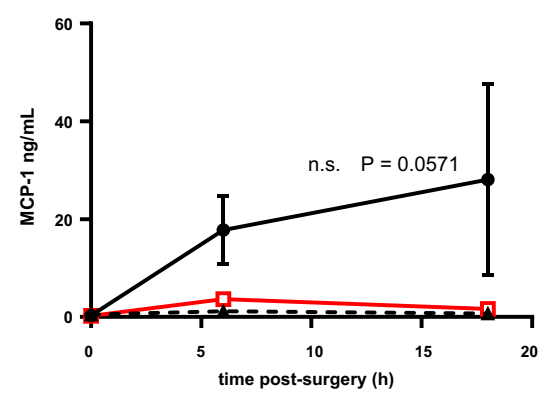

Figure 2 The cytokine response was attenuated in aged IRF3-KO septic mice. Aged WT and IRF3-KO mice were subject to CLP to induce sepsis, and a second group of aged WT mice were subject to sham surgery as a control. Graphs show pooled male and female data $(n=10 /$ group CLP, $n=5 /$ group sham) for (A) serum IL-6, (B) serum IL-12/ 23p40, (C) serum MCP-I. This cohort was split into male and female subgroups to show the impact of biological sex. Graphs show data for the male mice ( $n=5 / g r o u p ~ C L P$, $\mathrm{n}=3$ /group sham), including: (D) serum IL-6, (E) serum IL-12/23p40, (F) serum MCP-I and the female mice, ( $\mathrm{n}=5 /$ group $C L P, n=2 /$ group sham), including: (G) serum IL-6, (H) serum IL-12/23p40, and (I) serum MCP-I. P values show the results of Mann Whitney tests comparing the WT and IRF3-KO CLP groups at $6 \mathrm{~h}$ and I8h.

immune response to return to baseline has been documented in aged mice. ${ }^{10}$ Aged mice subject to CLP exhibit a higher degree of vascular oxidative damage and endothelial dysfunction, relative to young counterparts. $^{38}$ Furthermore, aged mice subject to CLP show increased coagulation coupled with reduced protein $\mathrm{C}$ activation, relative to young adult mice. ${ }^{39}$ All of these processes may contribute to the worse sepsis phenotype in aged vs young mice.
Although one cannot make a strict comparison between studies that were not performed concurrently, we note that the aged WT mice used in this study showed higher susceptibility to sepsis mortality (90\%; Figure 1A) compared to the adult WT mice subject to the same model in our recent report (38.5\%). ${ }^{20}$ Additionally, these aged WT mice showed increased disease score (Figure 1B), more severe hypothermia (Figure 1C) and higher levels of proinflammatory cytokines (Figure $2 \mathrm{~A}-\mathrm{C}$ ) as compared to the 


\section{Pooled}

AcK (serum 18h)
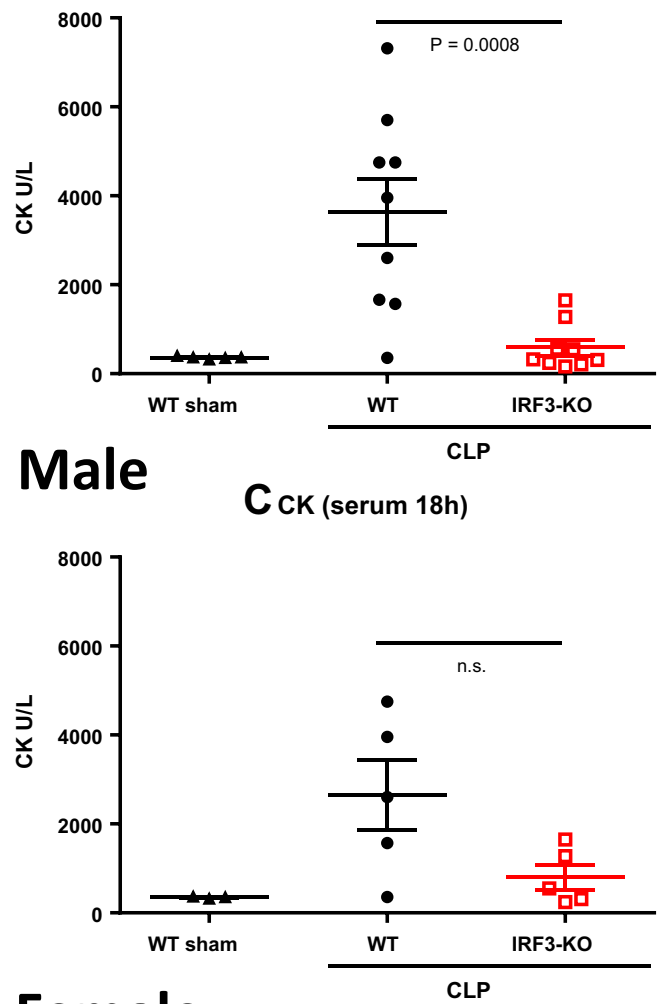

Female

EcK (Serum 18h)

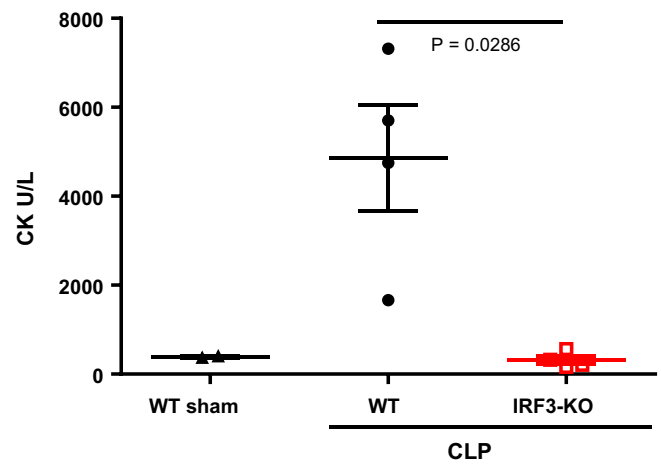

B ALT (serum 18h)

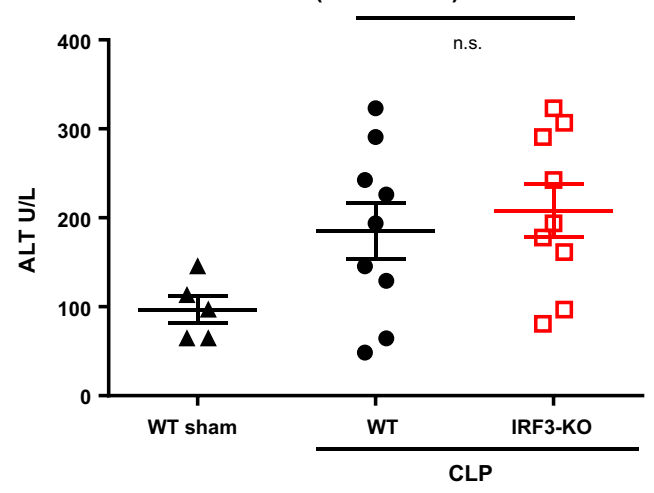

$D_{\text {ALT (serum 18h) }}$

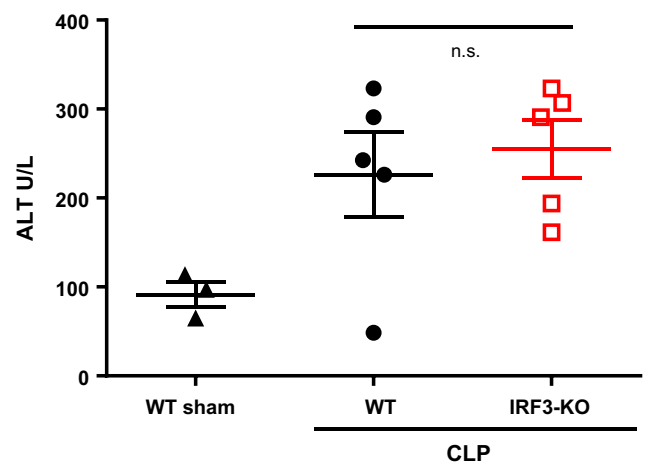

F ALT (Serum 18h)

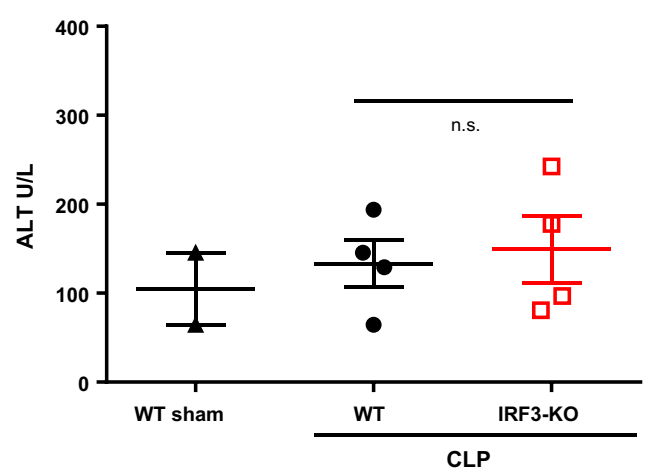

Figure 3 Serum CK and ALT levels in aged WT and IRF3-KO septic mice. Aged WT and IRF3-KO mice were subject to CLP to induce sepsis, and a second group of aged WT mice was subject to sham surgery as a control. At I8h post-surgery, serum CK levels were determined as a measure of heart/skeletal muscle damage, and serum ALT

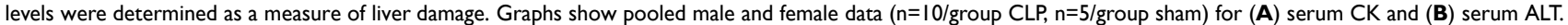

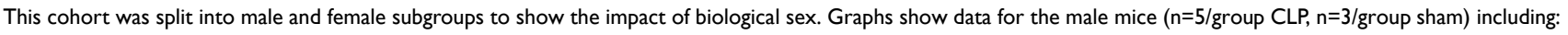
(C) serum CK and (D) serum ALT; and the female mice ( $n=5 /$ group CLP, $n=2 /$ group sham), including: (E) CK and (F) ALT. $P$ values show the results of Mann Whitney tests comparing the WT and IRF3-KO CLP groups.

adult mice from our recent report. ${ }^{20}$ Furthermore, in these aged WT mice, the levels of IL-6 increased from $6 \mathrm{~h}-18 \mathrm{~h}$ post-CLP (Figure 2A), whereas in the adult WT mice, IL-6 levels peaked at $6 \mathrm{~h}$ and then declined at $18 \mathrm{~h}^{20}$ These observations are consistent with the notion that sepsis is more severe in aged vs adult mice, associated with a more severe and sustained inflammatory cytokine response, as demonstrated in prior reports. ${ }^{3,10,12,36-39}$

IRF3 is a key transcription factor for IFN $\beta$ induction. ${ }^{42}$ IFN $\beta$ induces a range of Interferon Stimulated Genes that induce cellular resistance to infection and play important roles in cellular innate and adaptive immunity. IRF3 is 
important for the clearance of many viruses. However, we and others have shown that IRF3 also plays a significant, detrimental role in bacterial sepsis and related models of sterile, systemic inflammation in young adult mice. ${ }^{19,21,43-45}$ Additionally, we found that IFN $\beta$ is undetectable in the sera and peritoneal lavage of mice subject to CLP, ${ }^{20}$ suggesting that IRF3 may exacerbate sepsis via a different mechanism. In this study, we found that aged IRF3-KO mice are substantially protected from sepsis, relative to their aged WT counterparts, showing reduced mortality, lower disease score and attenuated hypothermia (Figure 1). Moreover, the aged IRF3-KO mice had substantially lower levels of inflammatory mediators (Figure 2) and CK in their serum (Figure 3), relative to the aged WT mice. These data provide novel information indicating that IRF3 plays a harmful role during sepsis in aged mice. In light of our prior reports, ${ }^{19,20}$ this study suggests that IRF3 performs the same function in sepsis, regardless of mouse age.

In addition to the effects of age, sepsis outcomes vary by gender. Most prior studies have reported worse sepsis outcomes in male vs female patients, ${ }^{46-50}$ and similar data were obtained in animal studies. ${ }^{40,51-53}$ However, these results were not unequivocal as some studies identified worse outcomes in females, ${ }^{54-56}$ or similar outcomes for both genders. ${ }^{57-59}$ More studies are needed to determine the effects of fluids and antibiotics on sepsis outcomes in males vs females. ${ }^{60}$ Our study highlights the impact of biological sex on sepsis in aged mice, and how this intersects with IRF3 deficiency. Aged WT female mice showed a trend towards greater survival and a lower disease score, as compared to aged WT male mice (Figure 1D, E, G and $\mathrm{H})$. However, when examining the mortality curve more closely, we noted that the females exhibited more rapid mortality during the acute phase of sepsis (days $0-3$ ) in comparison to males, while males exhibited a greater incidence of mortality in the chronic phase of sepsis (day 4 onward, Figure 1D and G). Paired with this finding, we observed more marked hypothermia in WT female vs male mice during the acute phase of sepsis (Figure $1 \mathrm{~F}$ and $\mathrm{I}$ ). Prior reports have demonstrated that animals which die in the acute phase of sepsis uniformly exhibit high levels of serum IL-6 predict acute mortality after CLP, while some animals that die in the chronic phase of sepsis exhibit immunosuppression. ${ }^{61,62}$ Therefore, it is perhaps not surprising that the WT females in our cohort exhibited a trend towards higher levels of serum cytokines at $6 \mathrm{~h}$ and $18 \mathrm{~h}$ post-CLP in comparison their male counterparts (Figure 2D-I).
Moreover, the difference between WT and IRF3-KO animal survival, disease score and surface temperature was more pronounced in aged female mice as compared to aged male mice. (Figure 1D-I). While CK levels were reduced in female IRF3-KO vs WT mice post-CLP, male IRF3-KO and WT mice showed similar CK levels postCLP. However, ALT levels were higher in male mice after CLP vs sham surgery (Figure 3D), while this was not the case in female mice (Figure 3F). As in our prior study examining young adult mice, ${ }^{19}$ IRF3-deficiency did not influence ALT levels in aged septic mice, regardless of gender (Figure 3B, D, and F). These data demonstrate that animal sex has a complex influence on sepsis, altering survival, cytokines and organ damage in specific ways, with an overall protective effect observed in females.

We speculate that the protection observed in females is likely due to direct and indirect effects of sex hormones on the immune response, as suggested by others. ${ }^{47,51,52}$ For example, estrogens can suppress lymphocyte apoptosis, while androgens promote apoptosis. ${ }^{63}$ Additionally, estrogens can enhance cellular and humoral adaptive immunity. ${ }^{63}$

We previously carried out studies to elucidate the upstream pattern recognition pathways that activate IRF3 during sepsis and explain its contribution to sepsis pathogenesis in young adult mice. We reported that both STING and TRIF contribute to sepsis pathogenesis, depending on the severity of the model and administration of antibiotics and fluids. ${ }^{24}$ Interestingly, however, mice lacking both STING and TRIF failed to recapitulate fully the protective phenotype observed in IRF3-KO mice, suggesting involvement of other molecules or pathways. ${ }^{24}$ Using bone marrow chimeras and adoptive transfer experiments, we showed that IRF3 predominantly influenced sepsis through its action in the stromal compartment, and had little effect within leukocytes. ${ }^{20}$ Stromal IRF3 indirectly altered monocyte IL-6 production, resulting in a canonical inflammatory network dominated by IL-6, Tumor Necrosis Factor (TNF) $\alpha$ and IL-1 $\beta$ in WT mice, while IRF3-KO mice showed a more sustained chemokine response. ${ }^{20}$ However, the precise stromal cell type wherein IRF3 exerts this function remains to be elucidated. Further mechanistic studies are warranted to determine how IRF3 influences sepsis in young and aged mice. Additionally, it will be important to determine the effects of IRF3 on immunosenescence, and how it affects animal survival in other models of sepsis (for example, sepsis induces by Staphylococcus aureus or Streptococcus pneumoniae) as 
well as 2-hit models of secondary infection and hemorrhage plus CLP.

This study on aged mice has two limitations that should be noted. First, we recently determined that the IRF3-KO mice in our animal colony were not fully congenic to $\mathrm{C} 57 \mathrm{BL} / 6 \mathrm{~J}$ and retained some gene alleles from the parent 129 .Svlm strain. ${ }^{20}$ This issue was not unique to our lab, as similar strain contamination was reported by another lab studying IRF3. ${ }^{43}$ To address this, we backcrossed the IRF3-KO mice to C57BL/6J mice for four generations to achieve $97 \%$ congenicity (verified by a genome scan). ${ }^{20}$ Using adult mice, we showed that the backcrossed IRF3-KO mice and non-backcrossed IRF3KO mice showed a virtually identical phenotype following CLP, and both were significantly protected from sepsis relative to WT C57BL/6J mice. ${ }^{20}$ Hence, the protected phenotype appears to stem from the IRF3-KO locus, rather than contaminating 129.Svlm alleles. Second, the sham controls used in this study were not perfectly age-matched to their CLP counterparts, which was due to issues with mouse availability.

\section{Conclusion}

In conclusion, our results demonstrate that IRF3 plays a detrimental role in sepsis in aged mice. We also determined that aged female mice exhibit more rapid acute sepsis mortality, while aged male mice are more prone to chronic sepsis mortality; furthermore, aged female mice had higher levels of serum cytokines and CK. Future study is warranted to continue to explore the role of IRF3 in sepsis. Ultimately, we hope to determine if IRF3 is a viable therapeutic target for sepsis treatment in people of all ages and genders.

\section{Abbreviations}

ALT, Alanine Aminotransferase; CK, Creatine Kinase; CLP, cecal ligation and puncture; IL, Interleukin; IRF3, Interferon Regulatory Factor 3; KO, knock-out; MCP, Macrophage Chemoattractant Protein; STING, Stimulator of Interferon Genes; TRIF, TIR-Domain Containing Adapter-inducing Interferon- $\beta$; WT, wild-type.

\section{Acknowledgments}

This study was supported by a grant from the El Paso Community Foundation awarded to WEW. We thank Chris Boehm, Irene Macias and the LARC staff of TTUHSC El Paso for their assistance with the study and care of our animals.

\section{Disclosure}

The authors reportls no conflicts of interest in this work.

\section{References}

1. Sepsis Alliance Critical Fact Sheet. www.sepsis.orgssepsis-factsheet-v2-4. Accessed January, 2018.

2. Rowe T, Araujo KL, Van Ness PH, Pisani MA, Juthani-Mehta M. Outcomes of older adults with sepsis at admission to an intensive care unit. Open Forum Infect Dis. 2016;3(1):ofw010.

3. Nacionales DC, Gentile LF, Vanzant E, et al. Aged mice are unable to mount an effective myeloid response to sepsis. J Immunol. 2014;192 (2):612-622.

4. Rudd KE, Johnson SC, Agesa KM, et al. Global, regional, and national sepsis incidence and mortality, 1990-2017: analysis for the Global Burden of Disease Study. Lancet. 2020;395(10219):200-211.

5. Iwashyna TJ, Ely EW, Smith DM, Langa KM. Long-term cognitive impairment and functional disability among survivors of severe sepsis. JAMA. 2010;304(16):1787-1794.

6. Mankowski RT, Anton SD, Ghita GL, et al. Older sepsis survivors suffer persistent disability burden and poor long-term survival. $J \mathrm{Am}$ Geriatr Soc. 2020;68(9):1962-1969.

7. Jones TK, Fuchs BD, Small DS, et al. Post-acute care use and hospital readmission after sepsis. Ann Am Thorac Soc. 2015;12 (6):904-913.

8. Opal SM, Girard TD, Ely EW. The immunopathogenesis of sepsis in elderly patients. Clin Infect Dis. 2005;41(Suppl 7):S504-512.

9. Piccirillo JF, Vlahiotis A, Barrett LB, Flood KL, Spitznagel EL, Steyerberg EW. The changing prevalence of comorbidity across the age spectrum. Crit Rev Oncol Hematol. 2008;67(2):124-132.

10. Gentile LF, Nacionales DC, Lopez MC, et al. Protective immunity and defects in the neonatal and elderly immune response to sepsis. $J$ Immunol. 2014;192(7):3156-3165. doi:10.4049/jimmunol.1301726

11. Stortz JA, Hollen MK, Nacionales DC, et al. Old mice demonstrate organ dysfunction as well as prolonged inflammation, immunosuppression, and weight loss in a modified surgical sepsis model. Crit Care Med. 2019;47(11):e919-e929.

12. Turnbull IR, Wlzorek JJ, Osborne D, Hotchkiss RS, Coopersmith CM, Buchman TG. Effects of age on mortality and antibiotic efficacy in cecal ligation and puncture. Shock. 2003;19(4):310-313.

13. Saito M, Inoue S, Yamashita K, Kakeji Y, Fukumoto T, Kotani J. IL15 improves aging-induced persistent $\mathrm{T}$ cell exhaustion in mouse models of repeated sepsis. Shock. 2020;53(2):228-235.

14. Takeda K, Akira S. TLR signaling pathways. Semin Immunol. 2004;16(1):3-9.

15. Cai X, Chiu Y-H, Chen Zhijian J. The cGAS-cGAMP-STING pathway of cytosolic DNA sensing and signaling. Mol Cell. 2014;54 (2):289-296.

16. Reikine S, Nguyen JB, Modis Y. Pattern recognition and signaling mechanisms of RIG-I and MDA5. Front Immunol. 2014;5:342.

17. Sabbah A, Chang TH, Harnack R, et al. Activation of innate immune antiviral responses by Nod2. Nat Immunol. 2009;10(10):1073-1080.

18. Hornung V, Ablasser A, Charrel-Dennis M, et al. AIM2 recognizes cytosolic dsDNA and forms a caspase-1-activating inflammasome with ASC. Nature. 2009;458(7237):514-518.

19. Walker WE, Bozzi AT, Goldstein DR. IRF3 contributes to sepsis pathogenesis in the mouse cecal ligation and puncture model. $J$ Leukoc Biol. 2012;92(6):1261-1268.

20. Heipertz EL, Harper J, Goswami DG, et al. IRF3 signaling within the mouse stroma influences sepsis pathogenesis. J Immunol. 2021;206 (2):398-409.

21. Walker WE, Booth CJ, Goldstein DR. TLR9 and IRF3 cooperate to induce a systemic inflammatory response in mice injected with liposome:DNA. Mol Ther. 2010;18(4):775-784. 
22. Petro TM. IFN regulatory factor 3 in health and disease. $J$ Immunol. 2020;205(8):1981-1989.

23. Mogensen TH. IRF and STAT transcription factors - from basic biology to roles in infection, protective immunity, and primary immunodeficiencies. Front Immunol. 2019;9:3047.

24. Heipertz EL, Harper J, Walker WE. STING and TRIF contribute to mouse sepsis, depending on severity of the disease model. Shock. 2017;47(5):621-631.

25. Albus U. Guide for the Care and Use of Laboratory Animals. 8th ed. London, England: SAGE Publications Sage UK; 2012.

26. Osuchowski MF, Ayala A, Bahrami S, et al. Minimum quality threshold in pre-clinical sepsis studies (MQTiPSS): an international expert consensus initiative for improvement of animal modeling in sepsis. Intensive Care Med Exp. 2018;6(1):26.

27. Walker WE. Methods to study the innate immune response to sepsis. Methods Mol Biol. 2018;1717:189-206.

28. Heipertz EL, Harper J, Lopez CA, Fikrig E, Hughes ME, Walker WE. Circadian rhythms influence the severity of sepsis in mice via a TLR2-dependent, leukocyte-intrinsic mechanism. $J$ Immunol. 2018;201(1):193-201.

29. Wardi G, Tainter CR, Ramnath VR, et al. Age-related incidence and outcomes of sepsis in California, 2008-2015. J Crit Care. 2021;62:212-217.

30. Kotfis K, Wittebole X, Jaschinski U, et al. A worldwide perspective of sepsis epidemiology and survival according to age: observational data from the ICON audit. J Crit Care. 2019;51:122-132.

31. Brakenridge SC, Efron PA, Stortz JA, et al. The impact of age on the innate immune response and outcomes after severe sepsis/septic shock in trauma and surgical intensive care unit patients. J Trauma Acute Care Surg. 2018;85(2):247-255.

32. Starr ME, Saito H. Sepsis in old age: review of human and animal studies. Aging Dis. 2014;5(2):126-136.

33. Yang Y, Yang KS, Hsann YM, Lim V, Ong BC. The effect of comorbidity and age on hospital mortality and length of stay in patients with sepsis. J Crit Care. 2010;25(3):398-405.

34. Martin GS, Mannino DM, Moss M. The effect of age on the development and outcome of adult sepsis. Crit Care Med. 2006;34(1):15-21.

35. Tran DD, Groeneveld AB, van der Meulen J, et al. Age, chronic disease, sepsis, organ system failure, and mortality in a medical intensive care unit. Crit Care Med. 1990;18(5):474-479.

36. Ren Z, Gay R, Thomas A, et al. Effect of age on susceptibility to Salmonella Typhimurium infection in C57BL/6 mice. $J$ Med Microbiol. 2009;58(Pt 12):1559-1567.

37. Das P, Panda SK, Agarwal B, et al. Novel chitohexaose analog protects young and aged mice from CLP induced polymicrobial sepsis. Sci Rep. 2019;9(1):2904.

38. Coletta C, Modis K, Olah G, et al. Endothelial dysfunction is a potential contributor to multiple organ failure and mortality in aged mice subjected to septic shock: preclinical studies in a murine model of cecal ligation and puncture. Crit Care. 2014;18(5):511.

39. Starr ME, Takahashi H, Okamura D, et al. Increased coagulation and suppressed generation of activated protein $\mathrm{C}$ in aged mice during intra-abdominal sepsis. Am J Physiol Heart Circ Physiol. 2015;308 (2):H83-91.

40. Drechsler S, Weixelbaumer K, Raeven P, et al. Relationship between age/gender-induced survival changes and the magnitude of inflammatory activation and organ dysfunction in post-traumatic sepsis. PLoS One. 2012;7(12):e51457.

41. Turnbull IR, Clark AT, Stromberg PE, et al. Effects of aging on the immunopathologic response to sepsis. Crit Care Med. 2009;37 (3):1018-1023.

42. Sato M, Tanaka N, Hata N, Oda E, Taniguchi T. Involvement of the IRF family transcription factor IRF-3 in virus-induced activation of the IFN-beta gene. FEBS Lett. 1998;425(1):112-116.
43. Yanai H, Chiba S, Hangai S, et al. Revisiting the role of IRF3 in inflammation and immunity by conditional and specifically targeted gene ablation in mice. Proc Natl Acad Sci USA. 2018;115(20):52535258.

44. Dang O, Navarro L, David M. Inhibition of lipopolysaccharide-induced interferon regulatory factor 3 activation and protection from septic shock by hydroxystilbenes. Shock. 2004;21(5):470-475.

45. Sakaguchi S, Negishi H, Asagiri M, et al. Essential role of IRF-3 in lipopolysaccharide-induced interferon-beta gene expression and endotoxin shock. Biochem Biophys Res Commun. 2003;306(4):860866.

46. Nasir N, Jamil B, Siddiqui S, Talat N, Khan FA, Hussain R. Mortality in sepsis and its relationship with gender. Pak J Med Sci. 2015;31 (5):1201-1206.

47. Zhang MQ, Macala KF, Fox-Robichaud A, Mendelson AA, Lalu MM. Sex- and gender-dependent differences in clinical and preclinical sepsis. Shock. 2021;56(2):178-187.

48. Cerceo E, Rachoin JS, Gaughan J, Weisberg L. Association of gender, age, and race on renal outcomes and mortality in patients with severe sepsis and septic shock. J Crit Care. 2021;61:52-56.

49. Adrie C, Azoulay E, Francais A, et al. Influence of gender on the outcome of severe sepsis: a reappraisal. Chest. 2007;132(6):17861793.

50. Schroder J, Kahlke V, Staubach KH, Zabel P, Stuber F. Gender differences in human sepsis. Arch Surg. 1998;133(11):1200-1205.

51. Bosch F, Angele MK, Chaudry IH. Gender differences in trauma, shock and sepsis. Mil Med Res. 2018;5(1):35.

52. Angele MK, Frantz MC, Chaudry IH. Gender and sex hormones influence the response to trauma and sepsis: potential therapeutic approaches. Clinics (Sao Paulo, Brazil). 2006;61(5):479-488.

53. Erikoğlu M, Sahin M, Ozer S, Avunduk MC. Effects of gender on the severity of sepsis. Surg Today. 2005;35(6):467-472.

54. Eachempati SR, Hydo L, Barie PS. Gender-based differences in outcome in patients with sepsis. Arch Surg. 1999;134(12):13421347.

55. Al Abbasi B, Torres P, Ramos-Tuarez F, et al. Implementation of the surviving sepsis campaign in patients with heart failure: genderspecific outcomes. Cureus. 2020;12(7):e9140.

56. Pietropaoli AP, Glance LG, Oakes D, Fisher SG. Gender differences in mortality in patients with severe sepsis or septic shock. Gend Med. 2010;7(5):422-437.

57. van Vught LA, Scicluna BP, Wiewel MA, et al. Association of gender with outcome and host response in critically ill sepsis patients. Crit Care Med. 2017;45(11):1854-1862.

58. Madsen TE, Simmons J, Choo EK, Portelli D, McGregor AJ, Napoli AM. The DISPARITY study: do gender differences exist in Surviving Sepsis Campaign resuscitation bundle completion, completion of individual bundle elements, or sepsis mortality? J Crit Care. 2014;29(3):473.e477-411.

59. Nachtigall I, Tafelski S, Rothbart A, et al. Gender-related outcome difference is related to course of sepsis on mixed ICUs: a prospective, observational clinical study. Crit Care. 2011;15(3):R151.

60. Zhang M, Montroy J, Sharma R, et al. The effects of biological sex on sepsis treatments in animal models: a systematic review and a narrative elaboration on sex- and gender-dependent differences in sepsis. Crit Care Explorat. 2021;3(6):e0433.

61. Remick DG, Bolgos GR, Siddiqui J, Shin J, Nemzek JA. Six at six: interleukin-6 measured $6 \mathrm{~h}$ after the initiation of sepsis predicts mortality over 3 days. Shock. 2002;17(6):463-467.

62. Chiswick EL, Mella JR, Bernardo J, Remick DG. Acute-phase deaths from murine polymicrobial sepsis are characterized by innate immune suppression rather than exhaustion. J Immunol. 2015;195 (8):3793-3802.

63. Klein SL. The effects of hormones on sex differences in infection: from genes to behavior. Neurosci Biobehav Rev. 2000;24(6):627-638. 


\section{Publish your work in this journal}

The Journal of Inflammation Research is an international, peerreviewed open-access journal that welcomes laboratory and clinica findings on the molecular basis, cell biology and pharmacology of inflammation including original research, reviews, symposium reports, hypothesis formation and commentaries on: acute/chronic inflammation; mediators of inflammation; cellular processes; molecular mechanisms; pharmacology and novel anti-inflammatory drugs; clinical conditions involving inflammation. The manuscript management system is completely online and includes a very quick and fair peerreview system. Visit http://www.dovepress.com/testimonials.php to read real quotes from published authors.

Submit your manuscript here: https://www.dovepress.com/journal-of-inflammation-research-journal 